\title{
Suitability of Drinking Water Sources from Nyaruzinga Wetland for Domestic Use in Bushenyi Municipality, Uganda
}

\author{
Lauben Muhangane*, John Bosco Nkurunungi, Jane Yatuha, Morgan Andama \\ Department of Biology, Faculty of Science, Mbarara University of Science and Technology, Mbarara, Uganda \\ Email: *muhangane16@gmail.com
}

How to cite this paper: Muhangane, L., Nkurunungi, J.B., Yatuha, J. and Andama, M. (2017) Suitability of Drinking Water Sources from Nyaruzinga Wetland for Domestic Use in Bushenyi Municipality, Uganda. Journal of Water Resource and Protection, 9, 1587-1611.

https://doi.org/10.4236/jwarp.2017.913100

Received: November 24, 2017

Accepted: December 25, 2017

Published: December 28, 2017

Copyright $\odot 2017$ by authors and Scientific Research Publishing Inc. This work is licensed under the Creative Commons Attribution International License (CC BY 4.0).

http://creativecommons.org/licenses/by/4.0/

cc) (i) Open Access

\begin{abstract}
This study determined the physico-chemical and bacterial quality of raw (borehole, springs, wells) and tap water sources from Nyaruzinga wetland for domestic use in Bushenyi Municipality. Forty samples of raw water were randomly collected from Kacuncu, Kyeitembe and Kikuba tributaries of the wetland and 40 samples of tap water generated from the same wetland were randomly drawn from Ishaka, Nyakabirizi and Central divisions of the Municipality. The samples were analyzed for apparent colour (AC), turbidity, electrical conductivity (EC), total hardness, $\mathrm{pH}$, total iron, residual alum, free residual chlorine and faecal coliforms using standard methods and their values compared with standard local UNBS (2008) and international WHO (2011) drinking water quality guidelines. The results showed that the mean $\mathrm{pH}$ of the protected spring below Kikuba Hill (5.21); the $\mathrm{AC}(\mathrm{Pt} / \mathrm{Co})$ of the borehole below Bweranyangi Junior School (17.28) and the surface well below Nyandozo Primary School (157.58) both situated in Kacuncu as well as the free residual chlorine $(\mathrm{mg} / \mathrm{L})$ in tap water at Kanyamabona Trading Centre, Ishaka division (0.192) were below the standard values of $\mathrm{WHO}(\mathrm{pH}, 6.0$ - 8.0; free residual chlorine, $0.2-0.5$ ) and UNBS (AC, <15). The electrical conductivity, EC $(\mu \mathrm{S} / \mathrm{cm})$ and total hardness $(\mathrm{mg} / \mathrm{L})$ of all the selected water sources were within the WHO standards (EC, $<1500$ (raw water), 0 - 300 (tap water); total hardness, $<1500$ (raw water), $<100$ (tap water)). The residual alum $(\mathrm{mg} / \mathrm{L})$ in all the tap water sources was also within the WHO guideline $(<0.2)$. On the other hand, the turbidity (27.38 NTUs) and total iron content $(0.32 \mathrm{mg} / \mathrm{L})$ of the surface well below Nyandozo Primary School were above WHO guidelines of $<15$ and $<0.3$ respectively. Furthermore, the mean faecal coliform counts (CFU/100 ml) in the surface well below Nyandozo Primary School (6.90) and the borehole below Bweranyangi Junior School (16.83) including the surface well below Kyeitembe Trading Centre (9.25) were above the WHO standard
\end{abstract}


faecal coliform count ( $\leq 3$ TCUs). Only the tap water at Kanyamabona Trading Centre recorded mean faecal coliform counts ( 0.25 TCUs) beyond the WHO standard (0 TCU). Hence the quality of tap water within the municipality tapped from Nyaruzinga wetland was generally better than the raw drinking water sources (borehole, springs, wells) from the same wetland. Thus the use of piped tap water by the urban inhabitants as opposed to raw water sources needs to be promoted to increase access to safe water.

\section{Keywords}

Bacterial Load, Bushenyi Municipality, Drinking Water Sources, Physico-Chemical Parameters

\section{Introduction}

Safe drinking water of adequate quantities is universally recognized as a basic human need and one of the most essential factors of human development as well as social and economic existence [1] [2]. However, millions of people in developing countries do not have access to adequate and safe water supply due to the increasing population and urbanization [3]. For instance, access to safe water remains a challenge in Africa with an overall estimate of $62 \%$ while access in rural areas is approximated at 47\% [4] [5]. The overall access in Uganda is about $66 \%$ with $42 \%$ in rural areas [6].

Uganda is highly endowed with water resources and wetlands covering 17\% (about $\left.40,000 \mathrm{~km}^{2}\right)$ of the total area of Uganda $\left(241,550 \mathrm{~km}^{2}\right)$ with wetlands constituting about $13 \%$ or $30,000 \mathrm{~km}^{2}$ of the country [7] [8]. With the rise in human population, and the subsequent demand for more resources to sustain their livelihood, wetlands and their benefits (e.g. water purification) are now under pressure both in rural and urban areas [8]. For example, it is estimated that about $75 \%$ of the wetland area in Uganda has been significantly affected by human activity, and about $13 \%$ is severely degraded [9]. Overall $67.8 \mathrm{~km}^{2}$ or $27.2 \%$ of the total wetland area in Bushenyi district has been modified for various activities, mostly dairy farming and cultivation. As a result, there are severe environmental problems of pollution of water resources in Uganda including Bushenyi [10]. However, addressing such current and future water pollution risks requires accurate data from consistent monitoring.

In the case of Bushenyi District, the safe water coverage is estimated at $85 \%$, with $93 \%$ in rural areas and 59\% in urban areas [11]. Bushenyi Municipality gets raw water from Nyaruzinga Swamp, which is first purified in a water treatment plant before supplying to the municipality [12]. However, the coverage of piped tap water within the municipality stands at $40 \%$ in Central, $20 \%$ in Ishaka and $20 \%$ in Nyakabirizi divisions with respective population densities of $496 / \mathrm{km}^{2}$, $881 / \mathrm{km}^{2}$ and $245 / \mathrm{km}^{2}$. Despite the above coverage, only $12 \%$ of the municipality's population is served by piped tap water due to reduced functionality of tap 
water points as a result of intermittent supply and affordability by consumers. As such, some individuals, communities and institutions have invested in their own water supply systems by constructing and using shallow wells (19\%), springs (67\%) and boreholes (3\%), which raises concern on the safety of these water sources for domestic use [11]. The percentage distribution of households without access to safe water in the divisions of Bushenyi municipality is as follows: Ishaka (21.3\%), Central (19.7\%) and Nyakabirizi (25.6\%) [13].

Unfortunately, to date, there is scanty literature on water quality characteristics of Nyaruzinga wetland, a source for treated tap water and raw water (surface wells, springs and boreholes) for domestic usage in Bushenyi Municipality. Thus there is need to analyze the quality of both raw water sources and piped tap water from the same wetland. Therefore, this study determined the physico-chemical parameters and faecal coliform content in raw water sources and piped tap water from Nyaruzinga wetland in Bushenyi Municipality.

\section{Materials and Methods}

\subsection{Location and Description of the Study Area}

Nyaruzinga wetland is located in Bushenyi Municipality, Bushenyi District, South-Western Uganda (Figure 1). The district lies between the coordinates; $0^{\circ}$ $\mathrm{N}$ and $0^{\circ} 46^{\prime} \mathrm{S}$ of the equator and $29^{\circ} 41^{\prime}$ East and $30^{\circ} 30^{\prime}$ West, with its headquarters located 340 kilometers from the capital city of Uganda, Kampala. Bushenyi district has a landscape comprising of hills, valleys (mostly occupied by wetlands which are drained by streams and rivers) and some stretches of plain land all located within an elevation of 910 - 2500 meters above sea level [14].

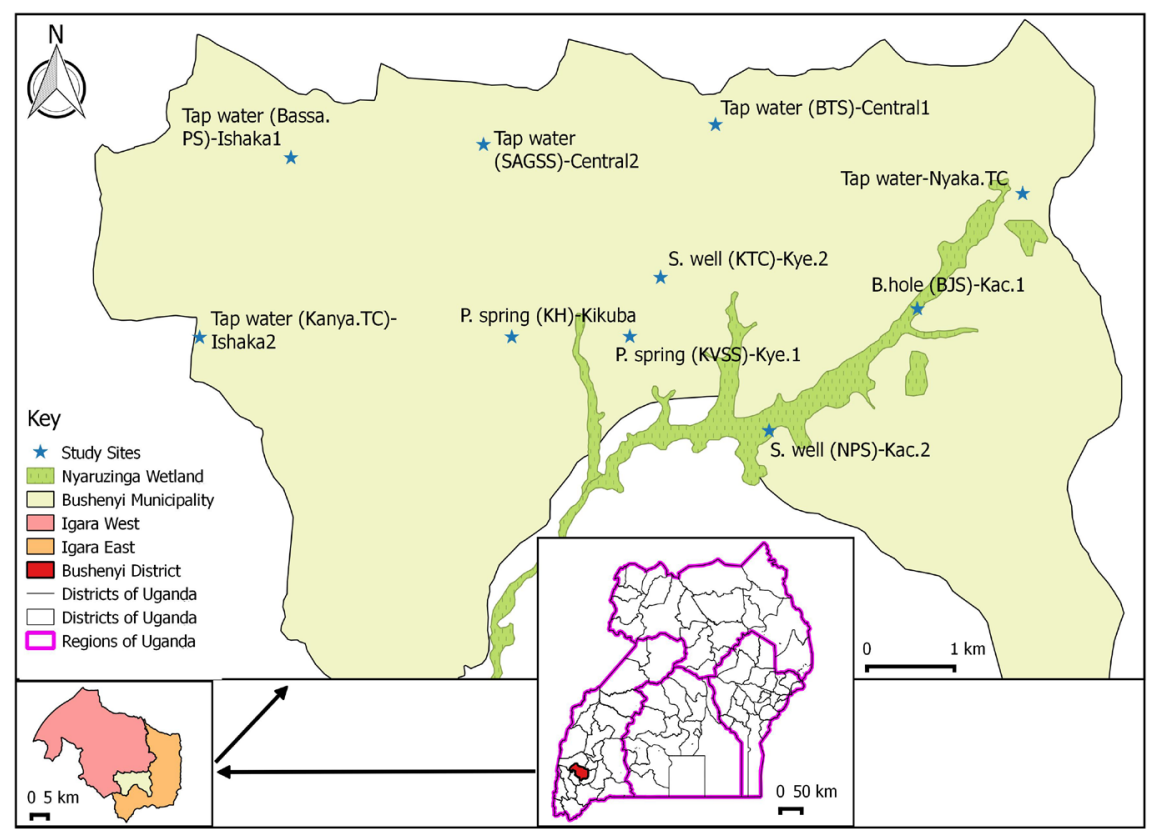

Figure 1. Map of Uganda showing the location of Bushenyi district, Nyaruzinga wetland and the study sites. 
The district receives an annual rainfall of 1500 - $2000 \mathrm{~mm}$ and has mean annual temperature ranging from $12.5^{\circ} \mathrm{C}$ to $30^{\circ} \mathrm{C}$. Greater Bushenyi has tropical rain forest vegetation of Kalinzu, and North Imaramagambo, and savannah woodlands and wetland vegetation which are widely distributed in the District. The natural forests of Kalinzu and Imaramagambo cover an estimated area of $784 \mathrm{~km}^{2}$. The district has a population of 251,400 who mainly engage in semiintensive agriculture, trade and commerce, transport, stone quarrying, sand mining, mineral mining, construction industry, tourism, and lumbering as their major economic activities [14].

Greater Bushenyi district has a total area of $4287 \mathrm{~km}^{2}$ of which $369 \mathrm{~km}^{2}$ is covered by open water. Wetlands cover an area of $250 \mathrm{~km}^{2}$ (5.8\% of the district) of which $132 \mathrm{~km}^{2}(52.8 \%)$ is permanent and $118 \mathrm{~km}^{2}(47.2 \%)$ is seasonal. The wetlands receive water mainly from rivers, precipitation and groundwater with Bushenyi having peak rains between March to April and September to December. The seasonal wetlands are usually flooded during the peak rainfall periods [8]. A total of $67.8 \mathrm{~km}^{2}$ of the wetland area (27.2\%) has been converted into pasture land for dairy cattle (about $36 \mathrm{~km}^{2}$ or $14 \%$ ) and for cultivation $\left(32 \mathrm{~km}^{2}\right.$ or $13 \%)[8]$.

Nyaruzinga wetland (Figure 1) is the only source of tap water that supplies the towns of Bushenyi, Nyakabirizi and Ishaka. The wetland is located about 2 $\mathrm{km}$ South of Bushenyi town in Bushenyi Municipality and has three major tributaries of Kyeitembe, Kacuncu and Kikuba streams. Nyaruzinga wetland is a tributary of Koga wetland system which drains into Lake Edward/George Drainage System through the Kasharara system. Nyaruzinga is a permanent converted wetland found in Mutara Sub-county occupying an area of $0.99 \mathrm{~km}^{2}$. It lies along a narrow valley (altitude, 1524 - $1585 \mathrm{~m}$ a.s.l.) surrounded by steep hills [8]. Flowing through the wetland is River Nyaruzinga which drains into Kateizi. The main plants in the wetland are sedges and papyrus dominated by Cyprus papyrus. Nyaruzinga wetland is used for livestock grazing, domestic water collection and fishing while the surrounding area is used for subsistence agriculture, livestock grazing and harvesting of plants for various uses. Heavy draining of the wetland for livestock grazing and crop growing are the major threats facing the wetland including soil erosion in the catchment. However, no conservation measures have been put in place to curb the wetland degradation [8].

\subsection{Geology of the Study Area}

Geology has an important bearing on drainage, geomorphologic processes and topography. The geology of Bushenyi district consists of the Precambrian rocks like shales, quarttzites, mudstones, schists, phyllites, gneisses and conglomerates which are rich in minerals [14]. The mineral ores in the district include beryl at Mutaka, columbite-tantalite in Jemubi and Kabira, copper-cobalt in Buhweju, gold in Kyamuhunga, quartz crystals (constituent of sandstone and granite 
rocks) in Buhweju, kaolin in Mutaka and Kibalya, pozzolana in Bunyaruguru volcanic fields. There are also potential mineral deposits of thorium in Buhweju, barites in Katoma hill, diamonds in volcanic fields in Bushenyi, feldspar in $\mathrm{Mu}$ taka and talc in Kyamuhunga [15]. These mineral ores influence some properties of the water bodies in Bushenyi District including Nyaruzinga wetland.

\subsection{Sampling Sites}

Five sites for raw water were purposively selected from the three tributaries of Nyaruzinga wetland (Figure 1) basing on the length of the tributaries. Two sites each were chosen along Kacuncu and Kyeitembe tributaries while one site was selected along Kikuba tributary. These sites were, Borehole below Bweranyangi Junior School in Kacuncu i.e., B.hole (BJS)-Kac. 1 (0.5540 ${ }^{\circ}$ S, 30.2081 ${ }^{\circ}$ E); Surface well below Nyandozo Primary School in Kacuncu, S. well (NPS)-Kac.2 (0.5663 ${ }^{\circ}$, $30.1928^{\circ} \mathrm{E}$ ); Protected spring below Kyeitembe Vocational Secondary School i.e., P. spring (KVSS)-Kye.1 $\left(0.5568^{\circ} \mathrm{S}, 30.1785^{\circ} \mathrm{E}\right)$; Surface well below Kyeitembe Trading Centre, S. well (KTC)-Kye. $2\left(0.5509^{\circ} \mathrm{S}, 30.1817^{\circ} \mathrm{E}\right)$ and Protected spring below Kikuba Hill i.e., P. spring (KH)-Kikuba $\left(0.5569^{\circ} \mathrm{S}, 30.1664^{\circ} \mathrm{E}\right)$.

Similarly, five tap water sources (Figure 1) were also purposively selected from the three divisions of Bushenyi Municipality basing on the population sizes of the divisions. Two tap water sources were each selected from Ishaka and Central divisions while one was chosen from Nyakabirizi Division. The sites included Tap water; near Bassajjabalaba Primary School in Ishaka, Tap water (Bassa. PS)-Ishaka1 $\left(0.5388^{\circ} \mathrm{S}, 30.1437^{\circ} \mathrm{E}\right)$; at Kanyamabona Trading Centre in Ishaka i.e., Tap water (Kanya. TC)-Ishaka2 $\left(0.5569^{\circ} \mathrm{S}, 30.1343^{\circ} \mathrm{E}\right)$; near Bushenyi Town School in Central, Tap water (BTS)-Central1 $\left(0.5354^{\circ} \mathrm{S}, 30.1873^{\circ} \mathrm{E}\right)$; near St. Agnes Girls Secondary School in Central i.e., Tap water (SAGSS)-Central2 $\left(0.5374^{\circ} \mathrm{S}, 30.1635^{\circ} \mathrm{E}\right)$ and at Nyakabirizi Trading Centre i.e., Tap water-Nyaka.TC $\left(0.5424^{\circ} \mathrm{S}, 30.2189^{\circ} \mathrm{E}\right)$.

\subsection{Water Collection}

Sample bottles were washed with dilute hydrochloric acid and rinsed with distilled water under aseptic conditions, to avoid contamination of water with other atmospheric bacteria. Using a sterilized dip sampling jug, water was scooped and transferred into the sample bottles for raw water while for tap water the sample bottle was placed under the tap and the tap was opened to run water into the bottle. Turbidity and $\mathrm{pH}$ were determined on site for raw water plus free residual chlorine for tap water and the bottle was then capped and transported in a transport box for microbial analysis at the Nyaruzinga water treatment plant. Forty samples were collected per study site within 40 days starting from $12^{\text {th }}$ September 2016 to $22^{\text {nd }}$ October, 2016.

\subsection{Laboratory Analysis}

The water samples were analysed according to Standard ISO Methods for Ex- 
amination of Water and Waste Water [16] [17] [18] and the Standards Operations Manual [19].

\subsection{1. $\mathrm{pH}$}

$\mathrm{pH}$ was measured using a $\mathrm{pH} / \mathrm{EC}$ multimeter. The wet cap of the electrode was removed and the electrode was rinsed with distilled water. The meter was switched on and the electrode dipped in the sample. The $\mathrm{pH}$ reading was left to stabilize and then recorded [19].

\subsubsection{Electrical Conductivity (EC)}

Electrical conductivity was also measured using a $\mathrm{pH} / \mathrm{EC}$ multimeter. The electrical conductivity electrode was removed from the wet cap and rinsed with distilled water. The MODE button was pressed to change from $\mathrm{pH}$ to EC measurement. The electrode was then dipped in the water sample and the meter left to stabilize and EC reading then recorded in $\mu \mathrm{s} / \mathrm{cm}[19]$.

\subsubsection{Turbidity}

Turbidity was measured using a turbidity meter. The turbidity meter was switched on. A volume of $10 \mathrm{ml}$ of the sample was put in the turbidity meter cell and inserted into the turbidity meter and the READ button pressed. The reading was left to stabilize and recorded in Nephlometeric Turbidity Units, (NTUs).

\subsubsection{Apparent Colour}

Apparent colour was measured using a Hach DR 5000 Spectrophotometer. The spectrophotometer was switched on and left to go through self check. A selection of the stored programs was made and scrolled to the parameter, colour. A volume of $10 \mathrm{mls}$ of distilled water was put into the sample cell and used to blank/zero the machine. The sample cell was then removed and emptied and 10 mls of the water sample put in it. The sample cell was put in the spectrophotometer and the option; READ was pressed. The value shown on the screen was recorded in TCUs.

\subsubsection{Total Hardness}

To measure Total Hardness, a burette was filled to the Zero mark with EDTA solution. A sample volume of $50 \mathrm{mls}$ was measured into a conical flask. Three drops of hardness buffer was added followed by $0.1 \mathrm{~g}$ of hardness indicator. The mixture was shaken and its colour turned pink. It was then titrated against EDTA solution until the colour turned from pink to blue/purple. The volume used was determined by subtracting initial burette reading from final burette reading and recorded. The value of total hardness in $\mathrm{mg} / \mathrm{l}$ was computed by multiplying the volume used by 20 .

\subsubsection{Total Iron}

Total iron was measured using a Hach DR 5000 spectrophotometer. A sample volume of $50 \mathrm{mls}$ was measured and diluted with distilled water to $100 \mathrm{mls}$. The 
solution was divided into two $50 \mathrm{ml}$ portions and put into two separate reaction bottles. One portion was kept as a blank and to the second portion was added an ample of ferrozine reagent, shaken and left for a 5 minute reaction time to lapse. The machine was zeroed using the blank. The ZERO button was pushed and the display showed $0.00 \mathrm{mg} / \mathrm{L} \mathrm{Fe}$. The prepared sample cell was cleaned and the prepared sample inserted into the cell holder. The READ button was then pushed and results showed in $\mathrm{mg} / \mathrm{L} \mathrm{Fe}$.

\subsubsection{Residual Alum}

Residual alum was also measured using a Hach DR 5000 Spectrophotometer. A sample volume of $50 \mathrm{mls}$ was measured into a reaction bottle. A pillow of ascorbic acid was added and shaken, followed by Awa 3 pillow reagent and also shaken. The solution was divided into two, $25 \mathrm{ml}$ portions. To one portion, a pillow of bleaching reagent was added for use as a blank to zero the machine. It was left for a reaction time of 15 minutes to elapse. After 15 minutes, the machine was blanked and the reacted sample value seen was multiplied by 2 to give the value of residual alum in $\mathrm{mg} / \mathrm{l}$.

\subsubsection{Free Residual Chlorine}

Free residual chlorine was measured using a chlorine meter. The chlorine meter was switched on and a sample volume of $10 \mathrm{mls}$ was put into a sample cell. The machine was adjusted to zero using the sample. In the same volume, $0.1 \mathrm{mg}$ of DPD powder reagent was added, shaken and measured to record the value in $\mathrm{mg} / \mathrm{l}$.

\subsubsection{Faecal Coliform Counts}

Faecal coliforms were measured using the membrane filtration method. Water was boiled in a sterilizing pan to sterilize the filtering gadgets. An absorbance pad was then put in a sterilizing dish and soaked with an enriched lactose culture media of about $2 \mathrm{mls}$. The sterilized filtering gadgets were then removed and fixed on the vacuum pump. The sterile filter membrane was put and $100 \mathrm{ml}$ of the sample was filtered through it. The membrane was then removed aseptically using forceps, put in the dish containing the lactose culture media and closed. The dish was then put in an incubator at an incubation temperature of $44.5^{\circ} \mathrm{C} \pm 0.2^{\circ} \mathrm{C}$ and the incubator was switched on for 24 hours. The incubator was then switched off from the power source. The sample was removed and a count of the number of blue colonies on the membrane was made and recorded in CFUs/ $100 \mathrm{ml}$.

\subsection{Data Analysis}

The descriptive statistics (minimum, maximum, standard deviation, mean, standard error of the mean) of the measured physico-chemical and biological parameters were tabulated. The variations of physico-chemical parameters and faecal coliform counts among the raw and tap water sources were established 
using one-way ANOVA (F) test while variations of the parameters between raw and tap water sources were ascertained by a z-test. Relationships between the measured water parameters (physico-chemical and faecal coliforms) were determined using Pearson correlation coefficient (r). Results were significant at 5\% level of significance. The data presentations and analyses were done using Microsoft Excel 2007 and SPSS 20.0 Statistical packages.

\section{Results and Discussion}

\subsection{Physico-Chemical Parameters in the Raw and Tap Water Sources in Bushenyi Municipality}

\subsection{1. $\mathrm{pH}$}

The $\mathrm{pH}$ is a measure of the hydrogen ion concentration in water [20]. The $\mathrm{pH}$ of water is important to living organisms as many biological processes occur only within a narrow $\mathrm{pH}$ range [21] [22]. There was low variability of $\mathrm{pH}$ within the samples of raw and tap water as well as the overall raw and tap water sources (CV; raw water, $0.94 \%$ - 9.98\%; tap, $0.63 \%-6.61 \%$; overall raw, $10.29 \%$; overall tap, 9.57\%) as shown in Tables 1-3. There was a significant variation $(\mathrm{p}<0.05)$ in the mean $\mathrm{pH}$ of the raw water sources with values ranging from 5.21 to 6.75 (Table 1). The highest $\mathrm{pH}$ was recorded in the surface well below Nyandozo Primary School and the lowest in the protected spring below Kikuba Hill. Similarly, $\mathrm{pH}$ varied significantly $(\mathrm{p}<0.05)$ among the tap water sites (Table 2). Tap water at Kanyamabona Trading Centre recorded the highest $\mathrm{pH}$ (7.06) while the least $\mathrm{pH}$ (5.72) was found in tap water at Nyakabirizi Trading Centre. On overall, raw water $(\mathrm{pH}=6.17)$ recorded significantly higher $\mathrm{pH}$ than tap water $(\mathrm{pH}=$ 6.03).

The mean $\mathrm{pH}$ ranges of most of the raw water sources fall within the WHO [23] standard limit of 6.0 - 8.0 apart from the protected spring below Kikuba Hill with mean pH below the WHO limit (Table 1). On the other hand, most of the raw water sources had mean $\mathrm{pH}$ below the UNBS [24] guideline of 6.5 - 8.5 except the surface well below Nyandozo Primary School (Table 1) with mean $\mathrm{pH}$ within the UNBS guideline. The $\mathrm{pH}$ ranges of tap water from all the sites (Table 2) fall within either the standard [24] or [23] values of 5.5 - 8.5 and 6.5 - 8.5 respectively. The overall mean $\mathrm{pH}$ of the raw water sources (Table 3) was also within the WHO guideline but below the UNBS standard. On the other hand, the overall mean $\mathrm{pH}$ of the tap water sources (Table 3) was within the UNBS guideline but below the WHO standard.

The mean $\mathrm{pH}$ values of the drinking water sources from Nyaruzinga wetland were slightly acidic possibly due to decomposition of organic material within the wetland forming acidic compounds which lower the $\mathrm{pH}$. According to Bot and Benites [25], the decomposition of organic wastes produces organic acids. The slight acidity of tap water is possibly attributed to the incomplete removal of the acidic compounds during the treatment process. Acidic $\mathrm{pH}$ was also recorded by Safari et al. [26] in most of the Nyaruzinga wetland water obtained near human activities as well as the treated tap water. 
Table 1. Descriptive statistics of water quality parameters for raw water from Nyaruzinga wetland.

\begin{tabular}{|c|c|c|c|c|c|c|c|c|c|c|}
\hline $\begin{array}{l}\text { Parameters } \\
(\mathrm{n}=40)\end{array}$ & Water source & Min. & Max. & SD & Mean \pm SE & CV (\%) & $\begin{array}{c}\text { P value for } \\
\text { F test }\end{array}$ & $\begin{array}{c}\text { Safari et al. } \\
\quad[26]\end{array}$ & $\begin{array}{c}\text { Standard } \\
{[24]}\end{array}$ & $\begin{array}{c}\text { Standard } \\
{[23]}\end{array}$ \\
\hline \multirow{5}{*}{$\mathrm{pH}$} & B.hole (BJS)-Kac.1 & 6.16 & 6.70 & 0.12 & $6.30 \pm 0.02$ & 1.83 & \multirow{5}{*}{$0.00^{*}$} & $7.27(\mathrm{~A})$ & \multirow{5}{*}{$6.5-8.5$} & \multirow{5}{*}{$6.0-8.0$} \\
\hline & S.well (NPS)-Kac.2 & 6.34 & 7.47 & 0.27 & $6.75 \pm 0.04$ & 4.03 & & 6.54 (B) & & \\
\hline & P.spring (KVSS)-Kye.1 & 5.60 & 6.81 & 0.43 & $6.19 \pm 0.07$ & 6.91 & & $5.67(\mathrm{C})$ & & \\
\hline & S.well (KTC)-Kye.2 & 5.77 & 7.32 & 0.64 & $6.42 \pm 0.10$ & 9.98 & & $5.68(\mathrm{D})$ & & \\
\hline & P.spring (KH)-Kikuba & 5.13 & 5.29 & 0.05 & $5.21 \pm 0.01$ & 0.94 & & $5.74(\mathrm{E})$ & & \\
\hline \multirow{5}{*}{$\begin{array}{c}\text { Electrical } \\
\text { Conductivity } \\
(\mu \mathrm{S} / \mathrm{cm})\end{array}$} & B.hole (BJS)-Kac.1 & 78.00 & 89.00 & 2.83 & $84.48 \pm 0.45$ & 3.35 & \multirow{5}{*}{$0.00^{*}$} & $614.04(\mathrm{~A})$ & \multirow{5}{*}{$<2500$} & \multirow{5}{*}{$<1500$} \\
\hline & S.well (NPS)-Kac.2 & 168.00 & 216.00 & 11.53 & $200.90 \pm 1.82$ & 5.74 & & $53.98(\mathrm{~B})$ & & \\
\hline & P.spring (KVSS)-Kye.1 & 60.00 & 79.00 & 5.51 & $69.63 \pm 0.87$ & 7.91 & & $53.32(\mathrm{C})$ & & \\
\hline & S.well (KTC)-Kye.2 & 103.00 & 142.00 & 11.93 & $124.63 \pm 1.89$ & 9.57 & & 35.75 (D) & & \\
\hline & P.spring (KH)-Kikuba & 46.00 & 79.00 & 11.49 & $65.08 \pm 1.82$ & 17.66 & & $42.35(\mathrm{E})$ & & \\
\hline \multirow{5}{*}{$\begin{array}{c}\text { Apparent } \\
\text { Colour }(\mathrm{Pt} / \mathrm{Co})\end{array}$} & B.hole (BJS)-Kac.1 & 13.00 & 20.00 & 1.87 & $17.28 \pm 0.30$ & 10.81 & \multirow{5}{*}{$0.00^{*}$} & $986.50(\mathrm{~A})$ & \multirow{5}{*}{$<15$} & \multirow{5}{*}{$<1500$} \\
\hline & S.well (NPS)-Kac.2 & 71.00 & 247.00 & 70.21 & $157.58 \pm 11.10$ & 44.56 & & $626.50(\mathrm{~B})$ & & \\
\hline & P.spring (KVSS)-Kye.1 & 0.00 & 10.00 & 2.82 & $3.28 \pm 0.45$ & 86.08 & & $236.83(\mathrm{C})$ & & \\
\hline & S.well (KTC)-Kye.2 & 3.00 & 8.00 & 1.43 & $4.95 \pm 0.23$ & 28.92 & & 385.33 (D) & & \\
\hline & P.spring (KH)-Kikuba & 0.00 & 11.00 & 3.26 & $4.03 \pm 0.52$ & 81.04 & & $415.33(\mathrm{E})$ & & \\
\hline \multirow{5}{*}{$\begin{array}{l}\text { Turbidity } \\
\text { (NTUs) }\end{array}$} & B.hole (BJS)-Kac.1 & 2.36 & 2.80 & 0.12 & $2.56 \pm 0.02$ & 4.65 & \multirow{5}{*}{$0.00^{*}$} & $236.17(\mathrm{~A})$ & \multirow{5}{*}{$<10$} & \multirow{5}{*}{$<15$} \\
\hline & S.well (NPS)-Kac.2 & 16.47 & 49.76 & 10.85 & $27.38 \pm 1.71$ & 39.61 & & 295.33 (B) & & \\
\hline & P.spring (KVSS)-Kye.1 & 0.80 & 1.29 & 0.15 & $1.09 \pm 0.02$ & 13.46 & & $165.17(\mathrm{C})$ & & \\
\hline & S.well (KTC)-Kye.2 & 0.30 & 0.51 & 0.06 & $0.40 \pm 0.01$ & 14.82 & & 191.17 (D) & & \\
\hline & P.spring (KH)-Kikuba & 1.47 & 2.15 & 0.23 & $1.80 \pm 0.04$ & 12.71 & & $211.33(\mathrm{E})$ & & \\
\hline \multirow{5}{*}{$\begin{array}{l}\text { Total Hardness } \\
(\mathrm{mg} / \mathrm{L})\end{array}$} & B.hole (BJS)-Kac.1 & 30.00 & 36.00 & 1.59 & $32.30 \pm 0.25$ & 4.92 & \multirow{5}{*}{$0.00^{*}$} & $43.61(\mathrm{~A})$ & \multirow{5}{*}{$<150$} & \multirow{5}{*}{$<1500$} \\
\hline & S.well (NPS)-Kac.2 & 14.00 & 21.00 & 1.75 & $17.23 \pm 0.28$ & 10.14 & & $16.21(\mathrm{~B})$ & & \\
\hline & P.spring (KVSS)-Kye.1 & 19.00 & 29.00 & 2.76 & $23.45 \pm 0.44$ & 11.79 & & $0.08(\mathrm{C})$ & & \\
\hline & S.well (KTC)-Kye.2 & 80.00 & 88.00 & 2.21 & $85.45 \pm 0.35$ & 2.58 & & 33.84 (D) & & \\
\hline & P.spring (KH)-Kikuba & 20.00 & 25.00 & 1.44 & $21.80 \pm 0.23$ & 6.59 & & $27.90(\mathrm{E})$ & & \\
\hline \multirow{5}{*}{$\begin{array}{l}\text { Total Iron } \\
(\mathrm{mg} / \mathrm{L})\end{array}$} & B.hole (BJS)-Kac.1 & 0.00 & 0.08 & 0.019 & $0.0394 \pm 0.003$ & 50.94 & \multirow{5}{*}{$0.00^{*}$} & \multirow{5}{*}{-} & \multirow{5}{*}{$<1$} & \multirow{5}{*}{$<0.3$} \\
\hline & S.well (NPS)-Kac.2 & 0.23 & 0.39 & 0.038 & $0.3183 \pm 0.006$ & 11.75 & & & & \\
\hline & P.spring (KVSS)-Kye.1 & 0.01 & 0.05 & 0.010 & $0.0343 \pm 0.002$ & 26.27 & & & & \\
\hline & S.well (KTC)-Kye.2 & 0.01 & 0.12 & 0.027 & $0.0363 \pm 0.004$ & 73.01 & & & & \\
\hline & P.spring (KH)-Kikuba & 0.00 & 0.06 & 0.013 & $0.0340 \pm 0.002$ & 41.23 & & & & \\
\hline \multirow{5}{*}{$\begin{array}{c}\text { Faecal } \\
\text { Coliforms } \\
(\mathrm{CFU} / 100 \mathrm{ml})\end{array}$} & B.hole (BJS)-Kac.1 & 0.00 & 65.00 & 25.19 & $16.83 \pm 3.98$ & 149.69 & & & & \\
\hline & S.well (NPS)-Kac.2 & 2.00 & 16.00 & 3.86 & $6.90 \pm 0.61$ & 55.87 & & & & \\
\hline & P.spring (KVSS)-Kye.1 & 0.00 & 9.00 & 3.06 & $1.95 \pm 0.48$ & 157.08 & $0.00^{*}$ & - & $<3$ & $<3$ \\
\hline & S.well (KTC)-Kye.2 & 0.00 & 24.00 & 8.28 & $9.25 \pm 1.31$ & 89.54 & & & & \\
\hline & P.spring $(\mathrm{KH})$-Kikuba & 0.00 & 2.00 & 0.67 & $0.40 \pm 0.11$ & 167.94 & & & & \\
\hline
\end{tabular}

*Significant difference; Min.-minimum; Max.-maximum; SD—standard deviation; SE-Standard error of the mean; CV—coefficient of variation; B.hole (BJS)-Kac.1-Borehole below Bweranyangi Junior School in Kacuncu; S.well (NPS)-Kac.2-Surface well below Nyandozo Primary School in Kacuncu; P.spring (KVSS)-Kye.1-Protected spring below Kyeitembe Vocational Secondary School; S.well (KTC)-Kye.2-Surface well below Kyeitembe Trading Centre; P.spring (KH)—Kikuba-Protected spring below Kikuba Hill; Direct wetland water source near: sewage discharge grounds (A), Fish ponds (B), Dip tank (C), raw water reservoir (D), car washing bay (E). 
Table 2. Descriptive statistics of water quality parameters for tap water in Ishaka, Central and Nyakabirizi divisions of Bushenyi Municipality.

\begin{tabular}{|c|c|c|c|c|c|c|c|c|c|c|}
\hline $\begin{array}{l}\text { Parameters } \\
(\mathrm{n}=40)\end{array}$ & Sites & $\min$. & $\max$ & SD & Mean \pm SE & $\mathrm{CV}(\%)$ & $\begin{array}{c}P \text { value } \\
\text { for } F \\
\text { test }\end{array}$ & $\begin{array}{l}\text { Safari } \\
\text { et al. } \\
{[26]}\end{array}$ & $\begin{array}{c}\text { Standard } \\
{[24]}\end{array}$ & $\begin{array}{c}\text { Standard } \\
\text { [23] }\end{array}$ \\
\hline \multirow{6}{*}{$\mathrm{pH}$} & Bassa.PS-Ishaka1 & 5.77 & 5.91 & 0.04 & $5.82 \pm 0.01$ & 0.63 & \multirow{5}{*}{$0.00^{*}$} & \multirow{5}{*}{6.03} & \multirow{5}{*}{$5.5-8.5$} & \multirow{5}{*}{$6.5-8.5$} \\
\hline & Kanya.TC-Ishaka2 & 5.72 & 7.52 & 0.47 & $7.06 \pm 0.07$ & 6.61 & & & & \\
\hline & BTS-Central1 & 5.60 & 6.39 & 0.27 & $5.84 \pm 0.04$ & 4.64 & & & & \\
\hline & SAGSS-Central2 & 5.58 & 5.82 & 0.07 & $5.68 \pm 0.01$ & 1.26 & & & & \\
\hline & Nyaka.TC & 5.59 & 5.88 & 0.10 & $5.72 \pm 0.02$ & 1.67 & & & & \\
\hline & Bassa.PS-Ishaka1 & 67.00 & 83.00 & 4.87 & $73.78 \pm 0.77$ & 6.60 & \multirow{5}{*}{$0.00^{*}$} & \multirow{5}{*}{176.17} & \multirow{5}{*}{$<1500$} & \multirow{5}{*}{$0-300$} \\
\hline \multirow{4}{*}{$\begin{array}{c}\text { Electrical } \\
\text { Conductivity } \\
(\mu \mathrm{S} / \mathrm{cm})\end{array}$} & Kanya.TC-Ishaka2 & 34.00 & 72.00 & 11.89 & $59.15 \pm 1.88$ & 20.10 & & & & \\
\hline & BTS-Central1 & 72.00 & 103.00 & 9.11 & $84.80 \pm 1.44$ & 10.75 & & & & \\
\hline & SAGSS-Central2 & 72.00 & 88.00 & 5.04 & $79.45 \pm 0.80$ & 6.35 & & & & \\
\hline & Nyaka.TC & 62.00 & 89.00 & 7.77 & $77.25 \pm 1.23$ & 10.06 & & & & \\
\hline \multirow{5}{*}{$\begin{array}{l}\text { Apparent Colour } \\
\text { (Pt/Co) }\end{array}$} & Bassa.PS-Ishaka1 & 0.00 & 11.00 & 3.04 & $5.60 \pm 0.48$ & 54.22 & \multirow{5}{*}{$0.00^{*}$} & \multirow{5}{*}{109.00} & \multirow{5}{*}{$<15$} & \multirow{5}{*}{$<300$} \\
\hline & Kanya.TC-Ishaka2 & 0.00 & 9.00 & 2.17 & $6.13 \pm 0.34$ & 35.50 & & & & \\
\hline & BTS-Central1 & 4.00 & 14.00 & 2.32 & $9.50 \pm 0.37$ & 24.43 & & & & \\
\hline & SAGSS-Central2 & 7.00 & 14.00 & 1.69 & $9.65 \pm 0.27$ & 17.49 & & & & \\
\hline & Nyaka.TC & 4.00 & 14.00 & 2.23 & $8.80 \pm 0.35$ & 25.37 & & & & \\
\hline \multirow{5}{*}{ Turbidity (NTUs) } & Bassa.PS-Ishaka1 & 0.83 & 1.99 & 0.40 & $1.44 \pm 0.06$ & 27.62 & \multirow{5}{*}{$0.00^{*}$} & \multirow{5}{*}{123.67} & \multirow{5}{*}{$<5.0$} & \multirow{5}{*}{$<5.0$} \\
\hline & Kanya.TC-Ishaka2 & 0.27 & 0.88 & 0.18 & $0.46 \pm 0.03$ & 40.20 & & & & \\
\hline & BTS-Central1 & 1.86 & 3.95 & 0.59 & $2.46 \pm 0.09$ & 23.94 & & & & \\
\hline & SAGSS-Central2 & 1.84 & 2.39 & 0.17 & $2.05 \pm 0.03$ & 8.13 & & & & \\
\hline & Nyaka.TC & 1.24 & 2.89 & 0.44 & $1.99 \pm 0.07$ & 22.05 & & & & \\
\hline \multirow{6}{*}{$\begin{array}{l}\text { Total Hardness } \\
\qquad(\mathrm{mg} / \mathrm{L})\end{array}$} & Bassa.PS-Ishaka1 & 24.00 & 39.00 & 3.90 & $33.05 \pm 0.62$ & 11.81 & \multirow{5}{*}{$0.00^{*}$} & \multirow{5}{*}{20.41} & \multirow{5}{*}{$<100$} & \multirow{5}{*}{$<100$} \\
\hline & Kanya.TC-Ishaka2 & 58.00 & 65.00 & 1.67 & $60.85 \pm 0.26$ & 2.75 & & & & \\
\hline & BTS-Centrall & 24.00 & 35.00 & 3.11 & $29.88 \pm 0.49$ & 10.40 & & & & \\
\hline & SAGSS-Central2 & 20.00 & 26.00 & 1.44 & $23.03 \pm 0.23$ & 6.26 & & & & \\
\hline & Nyaka.TC & 22.00 & 26.00 & 1.29 & $24.15 \pm 0.20$ & 5.35 & & & & \\
\hline & Bassa.PS-Ishaka1 & 0.060 & 0.230 & 0.043 & $0.136 \pm 0.007$ & 31.54 & & & & \\
\hline \multirow{4}{*}{ Total Iron (mg/L) } & Kanya.TC-Ishaka2 & 0.000 & 0.100 & 0.024 & $0.036 \pm 0.004$ & 66.48 & & & & \\
\hline & BTS-Central1 & 0.030 & 0.160 & 0.049 & $0.076 \pm 0.008$ & 63.75 & $0.00^{*}$ & - & $<0.2$ & $<0.3$ \\
\hline & SAGSS-Central2 & 0.080 & 0.190 & 0.032 & $0.111 \pm 0.005$ & 28.87 & & & & \\
\hline & Nyaka.TC & 0.040 & 0.140 & 0.024 & $0.096 \pm 0.004$ & 24.86 & & & & \\
\hline & Bassa.PS-Ishaka1 & 0.060 & 0.170 & 0.027 & $0.108 \pm 0.004$ & 24.83 & & & & \\
\hline & Kanya.TC-Ishaka2 & 0.000 & 0.160 & 0.037 & $0.092 \pm 0.006$ & 40.63 & & & & \\
\hline Residual Alum & BTS-Central1 & 0.070 & 0.160 & 0.026 & $0.113 \pm 0.004$ & 22.81 & $0.02^{*}$ & - & $<0.2$ & $<0.2$ \\
\hline & SAGSS-Central2 & 0.060 & 0.150 & 0.022 & $0.104 \pm 0.004$ & 21.39 & & & & \\
\hline & Nyaka.TC & 0.000 & 0.150 & 0.029 & $0.101 \pm 0.005$ & 28.78 & & & & \\
\hline
\end{tabular}




\section{Continued}

\begin{tabular}{|c|c|c|c|c|c|c|c|c|c|c|}
\hline \multirow{5}{*}{$\begin{array}{c}\text { Free Residual } \\
\text { Chlorine }(\mathrm{mg} / \mathrm{L})\end{array}$} & Bassa.PS-Ishaka1 & 0.210 & 0.360 & 0.038 & $0.275 \pm 0.006$ & 13.74 & \multirow{5}{*}{$0.00^{*}$} & \multirow{5}{*}{ - } & \multirow{5}{*}{$0.2-0.5$} & \multirow{5}{*}{$0.2-0.5$} \\
\hline & Kanya.TC-Ishaka2 & 0.060 & 0.320 & 0.075 & $0.192 \pm 0.012$ & 38.98 & & & & \\
\hline & BTS-Central1 & 0.200 & 0.300 & 0.033 & $0.248 \pm 0.005$ & 13.48 & & & & \\
\hline & SAGSS-Central2 & 0.230 & 0.310 & 0.023 & $0.257 \pm 0.004$ & 8.90 & & & & \\
\hline & Nyaka.TC & 0.200 & 0.290 & 0.026 & $0.249 \pm 0.004$ & 10.25 & & & & \\
\hline \multirow{5}{*}{$\begin{array}{c}\text { Faecal Coliforms } \\
\text { (CFU/100 ml) }\end{array}$} & Bassa.PS-Ishaka1 & 0.00 & 0.00 & 0.00 & $0.00 \pm 0.00$ & 0.00 & \multirow{5}{*}{-} & & \multirow{5}{*}{0} & \multirow{5}{*}{0} \\
\hline & Kanya.TC-Ishaka2 & 0.00 & 1.00 & 0.44 & $0.25 \pm 0.07$ & 175.41 & & & & \\
\hline & BTS-Central1 & 0.00 & 0.00 & 0.00 & $0.00 \pm 0.00$ & 0.00 & & - & & \\
\hline & SAGSS-Central2 & 0.00 & 0.00 & 0.00 & $0.00 \pm 0.00$ & 0.00 & & & & \\
\hline & Nyaka.TC & 0.00 & 0.00 & 0.00 & $0.00 \pm 0.00$ & 0.00 & & & & \\
\hline
\end{tabular}

*Significant difference; Min._-minimum; Max.—maximum; SD—standard deviation; SE-Standard error of the mean; CV—coefficient of variation; Bassa. PS-Bassajjabalaba Primary School; Kanya.TC_Kanyamabona Trading Centre; BTS-Bushenyi Town School; SAGSS-St.Agnes Girls Secondary School; Nyaka.TC-Nyakabirizi Trading Centre.

Table 3. Descriptive statistics for physico-chemical parameters between raw and tap water.

\begin{tabular}{|c|c|c|c|c|c|c|c|c|c|}
\hline $\begin{array}{l}\text { Parameter } \\
(\mathrm{n}=200)\end{array}$ & Water type & Min. & Max. & $\mathrm{SD}$ & Mean \pm SE & $\mathrm{CV}(\%)$ & $\begin{array}{c}P \text { value for } \\
Z \text { test }\end{array}$ & $\begin{array}{c}\text { Standard } \\
{[24]}\end{array}$ & Standard [23] \\
\hline \multirow{2}{*}{$\mathrm{pH}$} & Raw & 5.13 & 7.47 & 0.64 & $6.17 \pm 0.45$ & 10.29 & \multirow{2}{*}{$0.02^{*}$} & $6.5-8.5$ & $6.0-8.0$ \\
\hline & Tap & 5.58 & 7.52 & 0.58 & $6.03 \pm 0.04$ & 9.57 & & $5.5-8.5$ & $6.5-8.5$ \\
\hline \multirow{2}{*}{$\begin{array}{c}\mathrm{EC} \\
(\mu \mathrm{S} / \mathrm{cm})\end{array}$} & Raw & 46.00 & 216.00 & 51.52 & $108.94 \pm 3.64$ & 47.29 & \multirow{2}{*}{$0.00^{*}$} & $<2500$ & $<1500$ \\
\hline & Tap & 34.00 & 103.00 & 11.85 & $74.89 \pm 0.84$ & 15.83 & & $<1500$ & $0-300$ \\
\hline \multirow{2}{*}{$\begin{array}{c}\mathrm{AC} \\
(\mathrm{Pt} / \mathrm{Co})\end{array}$} & Raw & 0.00 & 247.00 & 68.01 & $37.42 \pm 4.81$ & 181.74 & \multirow{2}{*}{$0.00^{*}$} & $<15$ & $<1500$ \\
\hline & Tap & 0.00 & 14.00 & 2.88 & $7.94 \pm 0.20$ & 36.33 & & $<15$ & $<300$ \\
\hline \multirow{2}{*}{$\begin{array}{l}\text { Turbidity } \\
\text { (NTUs) }\end{array}$} & Raw & 0.30 & 49.76 & 11.47 & $6.64 \pm 0.81$ & 172.69 & \multirow{2}{*}{$0.00^{\star}$} & $<10$ & $<15$ \\
\hline & Tap & 0.27 & 3.95 & 0.79 & $1.68 \pm 0.06$ & 47.25 & & $<5.0$ & $<5.0$ \\
\hline \multirow{2}{*}{$\begin{array}{l}\text { Total Hardness } \\
\quad(\mathrm{mg} / \mathrm{L})\end{array}$} & Raw & 14.00 & 88.00 & 25.32 & $36.05 \pm 1.79$ & 70.25 & \multirow{2}{*}{0.36} & $<150$ & $<1500$ \\
\hline & Tap & 20.00 & 65.00 & 14.08 & $34.19 \pm 00$ & 41.19 & & $<100$ & $<100$ \\
\hline \multirow{2}{*}{$\begin{array}{l}\text { Total Iron } \\
(\mathrm{mg} / \mathrm{L})\end{array}$} & Raw & 0.00 & 0.39 & 0.12 & $0.0924 \pm 0.008$ & 124.22 & \multirow{2}{*}{0.84} & $<1$ & $<0.3$ \\
\hline & Tap & 0.00 & 0.23 & 0.05 & $0.0907 \pm 0.003$ & 53.54 & & $<0.2$ & $<0.3$ \\
\hline Faecal & Raw & 0.00 & 65.00 & 13.30 & $7.07 \pm 0.94$ & 188.26 & \multirow[b]{2}{*}{$0.00^{*}$} & $\leq 3$ & $\leq 3$ \\
\hline $\begin{array}{l}\text { Coliforms } \\
(\mathrm{CFU} / 100 \mathrm{ml})\end{array}$ & Tap & 0.00 & 1.00 & 0.22 & $0.05 \pm 0.02$ & 436.98 & & 0 & 0 \\
\hline
\end{tabular}

*Significant difference; Min.-minimum; Max.-maximum; SD—standard deviation; SE-Standard error of the mean; CV—coefficient of variation.

\subsubsection{Electrical Conductivity (EC)}

Electrical conductivity is the measure of the capability of water to transmit electric current and is a useful tool for assessment of the purity of water [27]. Conductivity does not directly affect human health. However it is measured to determine the rate of mineralization (existence of minerals such as potassium, calcium, and sodium) and also to estimate the amount of chemical reagents used to 
treat water [28] [29] [30] [31] [32].

The within sample variability of electrical conductivity in the raw (Table 1) and tap water (Table 2) sources were relatively low with CV ranging from 3.35\% - $17.66 \%$ (raw water) and $6.35 \%-20.10 \%$ (tap water). Furthermore, the variability of EC within samples of the overall tap water sources were relatively low (CV $=15.83 \%)$. However, the overall sample variability of EC in raw water sources was moderate $(\mathrm{CV}=47.29 \%)$. There was significant variation $(\mathrm{p}<0.05)$ in EC among the various raw and tap water sources (Table 1 and Table 2) and between the overall raw and tap water sources (Table 3). The surface well below Nyandozo Primary School also had the highest EC $(200.90 \mu \mathrm{S} / \mathrm{cm})$ while the Protected spring below Kikuba Hill recorded the least value $(65.08 \mu \mathrm{S} / \mathrm{cm})$.

The tap water near Bushenyi Town School recorded the highest EC $(84.80$ $\mu \mathrm{S} / \mathrm{cm})$ while the least EC $(59.15 \mu \mathrm{S} / \mathrm{cm})$ was found in tap water at Kanyamabona Trading Centre. On overall, raw water also recorded higher EC (108.94 $\mu \mathrm{S} / \mathrm{cm})$ than tap water $(\mathrm{EC}=74.89 \mu \mathrm{S} / \mathrm{cm})$. The EC values in each of the raw and tap water sources as well as the overall raw and tap water fall within the standard UNBS (raw water, $<2500 \mu \mathrm{S} / \mathrm{cm}$; tap water, $<1500 \mu \mathrm{S} / \mathrm{cm}$ ) and WHO (raw water, $<1500 \mu \mathrm{S} / \mathrm{cm}$; tap water, $0-300 \mu \mathrm{S} / \mathrm{cm}$ ) values. The EC of the groundwater sources (borehole and spring) were much lower than that of the surface wells. This signifies that there is very little dissolution in the groundwater, rapid ionexchange between the soil and water, or a poor and rather insoluble geologic rock and mineral types underlying Nyaruzinga wetland [3].

However the high EC in the surface well below Nyandozo Primary School could be associated with deposition of metal ions (e.g. iron etc) into the water through surface runoff despite some level of protection of the surface well. This is supported by the very high total iron in the surface well compared to other raw water sources (Table 1 ). Furthermore, there is an overall significant positive correlation between EC and Total iron content (Table 4) in all the water sources $(\mathrm{r}=0.75, \mathrm{p}<0.01)$. The EC in the drinking water sources in this study were also low compared to the EC of the direct wetland water obtained by Safari et al. [26] near sewage discharge grounds though the EC values of the drinking water sources were higher than the wetland water near fish ponds, dip tank, raw water reservoir and car washing bay. This implied that there was possibly an increased surface runoff of the metal ion into the wetland from human activities as the wetland does not receive any form of protection in its natural state. According to Mugyenyi and Mujurizi [8], there are no conservation measures put in place to prevent the degradation of Nyaruzinga wetland.

The electrical conductivity of the tap water sources is as a result of metal ions coming from the corrosion of the water pipes under acidic conditions (Table 2). Acidic water can leach metals from pipes and fixtures, such as copper, lead, zinc, iron and manganese [28] [33]. In addition, there were also significant $(\mathrm{p}<0.01)$ negative and positive correlations of Total iron with $\mathrm{pH}(\mathrm{r}=-0.45)$ and $\mathrm{EC}(\mathrm{r}=$ $0.34)$ respectively for tap water (Table 4 ). These therefore signify that corrosion 
of metal pipes under acidic conditions released iron which resulted into high electrical conductivity. Furthermore, conditions were also favourable for corrosion in most of the tap water sources which had $\mathrm{pH}$ less than 6 except the tap water at Kanyamabona Trading Centre ( $\mathrm{pH}$ 7.06). According to WHO [33], corrosion is prevented at a $\mathrm{pH}$ value of 6.5 or higher for drinking water. A previous study by Yasin et al. [34], also attributed the high conductivity recorded in

Table 4. Pearson correlation ( $\mathrm{r}$ ) matrix between the measured parameters in all the water sources $(\mathrm{n}=400)$ and tap water sources $(\mathrm{n}=200)$.

\begin{tabular}{|c|c|c|c|c|c|c|c|c|c|c|}
\hline Parameters & & $\mathrm{pH}$ & EC & $\mathrm{AC}$ & Turbidity & $\begin{array}{c}\text { Total } \\
\text { Hardness }\end{array}$ & $\begin{array}{l}\text { Total } \\
\text { Iron }\end{array}$ & $\begin{array}{l}\text { Residual } \\
\text { Alum }\end{array}$ & $\begin{array}{l}\text { Free Residual } \\
\text { Chlorine }\end{array}$ & $\begin{array}{c}\text { Faecal } \\
\text { Coliforms }\end{array}$ \\
\hline \multirow{3}{*}{$\mathrm{pH}$} & $\mathrm{r}$ & & $0.34^{* *}$ & $0.33^{\star *}$ & $0.28^{\star *}$ & $0.41^{\star *}$ & $0.19^{* *}$ & - & - & $0.26^{* *}$ \\
\hline & $\mathrm{p}$ & & 0.00 & 0.00 & 0.00 & 0.00 & 0.00 & - & - & 0.00 \\
\hline & $\mathrm{n}$ & & 400 & 400 & 400 & 400 & 400 & - & - & 400 \\
\hline \multirow{3}{*}{$\mathrm{EC}$} & $\mathrm{r}$ & $-0.58^{\star *}$ & & $0.80^{* *}$ & $0.81^{\star *}$ & -0.02 & $0.75^{\star *}$ & - & - & $0.23^{* *}$ \\
\hline & $\mathrm{p}$ & 0.00 & & 0.00 & 0.00 & 0.71 & 0.00 & - & - & 0.00 \\
\hline & $\mathrm{n}$ & 200 & & 400 & 400 & 400 & 400 & - & - & 400 \\
\hline \multirow{3}{*}{$\mathrm{AC}$} & $\mathrm{r}$ & $-0.37^{* *}$ & $0.27^{\star *}$ & & $0.97^{* *}$ & $-0.27^{\star *}$ & $0.77^{\star *}$ & - & - & $0.13^{*}$ \\
\hline & $\mathrm{p}$ & 0.00 & 0.00 & & 0.00 & 0.00 & 0.00 & - & - & 0.012 \\
\hline & $\mathrm{n}$ & 200 & 200 & & 400 & 400 & 400 & - & - & 400 \\
\hline \multirow{3}{*}{ Turbidity } & $\mathrm{r}$ & $-0.72^{\star *}$ & $0.56^{* *}$ & $0.66^{* *}$ & & $-0.32^{\star *}$ & $0.78^{* *}$ & - & - & $0.11^{*}$ \\
\hline & $\mathrm{p}$ & 0.00 & 0.00 & 0.00 & & 0.00 & 0.00 & - & - & 0.027 \\
\hline & $\mathrm{n}$ & 200 & 200 & 200 & & 400 & 400 & - & - & 400 \\
\hline \multirow{3}{*}{$\begin{array}{c}\text { Total } \\
\text { Hardness }\end{array}$} & $\mathrm{r}$ & $0.88^{\star *}$ & $-0.67^{\star *}$ & $-0.38^{\star *}$ & $-0.77^{\star \star}$ & & $-0.38^{\star *}$ & - & - & $0.13^{\star *}$ \\
\hline & $\mathrm{p}$ & 0.00 & 0.00 & 0.00 & 0.00 & & 0.00 & - & - & 0.008 \\
\hline & $\mathrm{n}$ & 200 & 200 & 200 & 200 & & 400 & - & - & 400 \\
\hline \multirow{3}{*}{ Total Iron } & $\mathrm{r}$ & $-0.45^{\star *}$ & $0.34^{* *}$ & -0.002 & $0.28^{\star *}$ & $-0.51^{\star *}$ & & - & - & 0.03 \\
\hline & $\mathrm{p}$ & 0.00 & 0.00 & 0.975 & 0.00 & 0.00 & & - & - & 0.62 \\
\hline & $\mathrm{n}$ & 200 & 200 & 200 & 200 & 200 & & - & - & 400 \\
\hline \multirow{3}{*}{$\begin{array}{l}\text { Residual } \\
\text { Alum }\end{array}$} & $\mathrm{r}$ & $-0.22^{\star *}$ & -0.02 & $0.16^{*}$ & $0.25^{\star *}$ & $-0.15^{*}$ & 0.112 & & - & - \\
\hline & $\mathrm{p}$ & 0.002 & 0.77 & 0.03 & 0.00 & 0.03 & 0.114 & & - & - \\
\hline & $\mathrm{n}$ & 200 & 200 & 200 & 200 & 200 & 200 & & - & - \\
\hline \multirow{3}{*}{$\begin{array}{l}\text { Free Resid- } \\
\text { ual Chlorine }\end{array}$} & $\mathrm{r}$ & $-0.56^{* *}$ & $0.14^{*}$ & 0.08 & $0.35^{\star *}$ & $-0.47^{\star \star}$ & $0.51^{* *}$ & 0.14 & & - \\
\hline & $\mathrm{p}$ & 0.00 & 0.04 & 0.29 & 0.00 & 0.00 & 0.00 & 0.06 & & - \\
\hline & $\mathrm{n}$ & 200 & 200 & 200 & 200 & 200 & 200 & 200 & & - \\
\hline \multirow{3}{*}{$\begin{array}{c}\text { Faecal } \\
\text { Coliforms }\end{array}$} & $\mathrm{r}$ & $0.46^{* *}$ & -0.13 & -0.099 & $-0.34^{\star \star}$ & $0.46^{\star *}$ & $-0.26^{\star *}$ & $-0.16^{*}$ & $-0.75^{\star \star}$ & \\
\hline & $\mathrm{p}$ & 0.00 & 0.06 & 0.165 & 0.00 & 0.00 & 0.00 & 0.02 & 0.00 & \\
\hline & $\mathrm{n}$ & 200 & 200 & 200 & 200 & 200 & 200 & 200 & 200 & \\
\hline
\end{tabular}

${ }^{*}$ Correlation is significant at the 0.01 level (2-tailed). ${ }^{*}$ Correlation is significant at the 0.05 level (2-tailed). 
tap water sources to the corrosion of metals which led to the accumulation of heavy metals. The very high EC of the tap water $(176.17 \mu \mathrm{S} / \mathrm{cm})$ obtained by Safari et al. [26] compared to EC values of the tap water sources in this current study (Table 2) can be associated with increased corrosion of metal pipes which were exclusively used for supply of tap water in the past years. However, there has been a replacement of most of the metal pipes with plastic pipes in the recent years.

\subsubsection{Apparent Colour (AC)}

Drinking-water should ideally have no visible colour. Nevertheless, colour in drinking-water is usually due to the presence of coloured organic matter (primarily humic and fulvic acids) associated with the humus fraction of soil as well as the presence of iron and other metals, either as natural impurities or as corrosion products [23]. However, the most common cause of water color is the presence of minerals mainly iron and manganese, eroded soil followed by algal growth [35]. There were highly variable values of apparent colour of water within samples for the raw water sources (CV, 10.81\% - 86.08\%), tap water sources (CV, $17.49 \%-54.22 \%)$ and the overall raw $(\mathrm{CV}=181.74 \%)$ and tap water $(\mathrm{CV}=36.33 \%)$ sources. The apparent colour of water significantly $(\mathrm{p}<0.05)$ varied among the raw and tap water sources and between the raw and tap water sources on overall. The surface well below Nyandozo Primary School had the highest apparent colour (157.58 Pt/Co) while the protected spring below Kyeitembe Vocational Secondary School had the least apparent colour (3.28 Pt/Co). Tap water near St. Agnes Girls Secondary School had the highest apparent colour (9.65 Pt/Co) and that near Bassajjabalaba Primary School had the least (5.60 $\mathrm{Pt} / \mathrm{Co})$. On overall, raw water registered a higher apparent colour (37.42 $\mathrm{Pt} / \mathrm{Co})$ than tap water $(7.94 \mathrm{Pt} / \mathrm{Co})$.

The apparent colour (AC) of the protected spring below Kyeitembe Vocational Secondary School, Surface well below Kyeitembe Trading Centre and the protected spring below Kikuba Hill were within the UNBS standard of $<15 \mathrm{Pt} / \mathrm{Co}$ (Table 1). On the other hand, the AC of the Borehole below Bweranyangi Junior School and the Surface well below Nyandozo Primary School were above the UNBS guideline value. However, the apparent colour of all the raw water sources were within the WHO guideline of $<1500 \mathrm{Pt} / \mathrm{Co}$. Similarly the apparent colour of all the tap water sources were within the UNBS $(<15 \mathrm{Pt} / \mathrm{Co})$ and WHO $(<300$ $\mathrm{Pt} / \mathrm{Co}$ ) standard values (Table 2). The raw water sources on overall had apparent colour beyond UNBS guideline value but within the WHO standard value. On the other hand, the tap water sources on overall recorded low apparent colour but within both UNBS and WHO guideline values.

The relatively high apparent colour of the surface well below Nyandozo Primary School as opposed to the other water sources is due to surface runoff of some particulate matter especially from gardens within the watershed causing high turbidity and total iron content in the water (Table 1). This is supported by the overall significant positive correlation of apparent colour with turbidity $(r=$ 
0.97, $\mathrm{p}<0.01)$ and total iron $(\mathrm{r}=0.77, \mathrm{p}<0.01)$ as shown in Table 4. According to Walakira [35], water color is attributed to the presence of minerals mainly iron as well as eroded soil. The low apparent colour of the drinking water sources from Nyaruzinga wetland signifies low concentration of total suspended and dissolved solids possibly due to some level of protection of the water sources. However, the high apparent colour of the water obtained directly from the wetland in a previous study [26] is associated with siltation from the degraded surrounding areas by human activities. According to Mugyenyi and $\mathrm{Mu}$ jurizi [8], Nyaruzinga wetland has been threatened mainly by the increased rate of conversion and siltation resulting from soil erosion from the surrounding agricultural activities and settlement.

The low apparent colour of water from the protected spring is due to filtration of suspended materials as the water percolates through the soil while the lower apparent colour of the tap water compared to raw water is due to the removal of most of the particles responsible for colour in water during the water treatment process. Hence the compliance of the apparent colour of the tap water with WHO standards reflects efficiency in the water treatment process by National Water and Sewerage Corporation. However, the higher apparent colour of the tap water (109.00 Pt/Co) reported by Safari et al. [26] than the apparent colour of the tap water sources in the present study (Table 2) is also attributable to corrosion under rampart use of metal pipes in the supply of water in the past years as opposed to the recent years where most of the metal pipes have been replaced with plastic pipes.

\subsubsection{Turbidity}

Turbidity is the measure of the clarity or cloudiness of water [21]. Turbidity in water is caused by the presence of suspended matter such as clay, silt, finely divided organic and inorganic matter, plankton, and other microscopic organisms [35]. Water turbidity had relatively low variability within samples among the raw (CV, 4.65\% - 39.61\%) and tap water sources (CV, 8.13\% - 40.20\%). However the within sample variability was moderately high for tap water $(\mathrm{CV}=47.35 \%)$ and very high for raw water $(\mathrm{CV}=172.69 \%)$ on overall. The turbidity of water from the various raw and tap water sources as well as the overall raw and tap water were significantly $(\mathrm{p}<0.05)$ different from one another. The surface well below Nyandozo Primary School had the highest turbidity (27.38 NTUs) while the surface well below Kyeitembe Trading Centre recorded the least turbidity (0.40 NTUs). The tap water near Bushenyi Town School was the most turbid (2.46 NTUs) whereas the tap water at Kanyamabona Trading Centre was the least turbid (0.46 NTUs). On overall, raw water sources (6.64 NTUs) were also more turbid than tap water sources (1.68 NTUs).

The turbidity of the Borehole below Bweranyangi Junior School, Protected spring below Kyeitembe Vocational Secondary School, Surface well below Kyeitembe Trading Centre and the protected spring below Kikuba Hill were within both UNBS (<10 NTUs) and WHO (<15 NTUs) guideline values for raw water. 
However, the surface well below Nyandozo Primary School recorded turbidity beyond the UNBS and WHO standard values. On the other hand, the turbidity of the tap water sources were all within the UNBS and WHO guideline of $<5$ NTUs. Similarly, the overall raw water sources registered turbidity (6.64 NTUs) in the range of UNBS and WHO standard values. The overall turbidity of the tap water sources (1.68 NTUs) was also within the UNBS and WHO standards for tap water.

Similarly, the high turbidity of the surface wells and the direct wetland water obtained by Safari et al. [26] is attributed to siltation from the increased rates of soil erosion within the catchment. The generally higher turbidity of the surface wells than other water sources and the least turbidity of the tap water sources are consistent with the findings of Yasin et al. [34] who obtained higher mean turbidity in unprotected wells compared to tap water. Nevertheless the very high turbidity of the tap water [26] compared to the turbidity values of the tap water sources in this current study also points to increased corrosion under the sole use of metal pipes in supply of water in the past years. However, most of the metal pipes have now being replaced by plastic pipes. The significant positive correlation of turbidity and total iron $(\mathrm{r}=0.28, \mathrm{p}<0.01)$ in tap water (Table 4) supports the fact that turbidity of tap water increases under corrosion of metallic pipes which release iron and other metals.

According to Rahmanian et al. [28], turbidity is also related to the content of disease causing organisms in water originating from soil runoff. The overall significant positive correlation between turbidity and faecal coliforms $(r=0.11, \mathrm{p}<$ $0.05)$ justify the surface runoff of soil particles with faecal coliforms into the surface wells. Mugyenyi and Mujurizi [8] reported that most fertile soils have been eroded down to the valleys (wetlands) due to the hilly terrain and poor farming methods in Bushenyi.

\subsubsection{Total Hardness}

Hardness of water is attributed to mainly the bicarbonates, carbonates, sulphates and chlorides of calcium and magnesium [20]. Total hardness of the raw and tap water sources exhibited low variability within the samples i.e. raw water $(\mathrm{CV}=$ $2.58 \%-11.79 \%)$, tap water (CV $=2.75 \%-11.81 \%)$. However the within sample variability for the overall tap water sources was moderate $(\mathrm{CV}=41.19 \%)$ while that for the raw water sources was high $(\mathrm{CV}=70.25 \%)$. There were significant differences $(\mathrm{p}<0.05)$ in the total hardness among the various raw and tap water sources. The surface well below Kyeitembe Trading Centre had the highest total hardness $(85.45 \mathrm{mg} / \mathrm{L})$ while that below Nyandozo Primary School had the least hardness $(17.23 \mathrm{mg} / \mathrm{L})$. On the other hand, the tap water at Kanyamabona Trading Centre recorded the highest total hardness $(60.85 \mathrm{mg} / \mathrm{L})$ with the least hardness $(23.03 \mathrm{mg} / \mathrm{L})$ being registered in tap water near St. Agnes Girls Secondary School. However there was no significant variation $(\mathrm{p}>0.05)$ in the overall total hardness of the raw and tap water sources though raw water (36.05 $\mathrm{mg} / \mathrm{L})$ recorded slightly higher total hardness than tap water $(34.19 \mathrm{mg} / \mathrm{L})$ on 
overall.

The mean total hardness of the different raw and tap water sources including the overall raw and tap water sources were within the UNBS and WHO guideline values for raw water (UNBS, $<150 \mathrm{mg} / \mathrm{L}$ and $\mathrm{WHO},<1500 \mathrm{mg} / \mathrm{L}$ ) and tap water (UNBS and WHO, <100 mg/L) (Tables 1-3). The mean total hardness of all the raw drinking and tap water sources as well as the direct wetland water and tap water [26], were within the WHO recommended range. However, on the contrary, the groundwater sources (boreholes and springs) which are in contact with geologic formations for longer periods of time had lower total hardness compared to the surface well. This is probably also attributed to very low dissolution in the groundwater, rapid ion-exchange between the soil and water and a poor and insoluble $\mathrm{Ca}$ and $\mathrm{Mg}$ containing geologic rock and mineral types underlying Nyaruzinga wetland [3]. Hence there is possibility of external sources of calcium and magnesium into the surface wells through surface runoff culminating into the higher total hardness. The low total hardness of tap water shows efficiency in removing metal ions in the water treatment process.

Basing on Durfor and Becker [36] categorization of hardness (mg/L) of water ( 0 - 60, soft; 61 - 120, moderately hard; $121-180$, hard; $>180$, very hard), most of the raw and tap water sources (Table 1 and Table 2) are soft except the surface well below Kyeitembe Trading Centre and tap water at Kanyamabona Trading Centre which are moderately hard. Very hard water is not desirable for many domestic uses as it leaves a scaly deposit on the inside of pipes, boilers, and tanks [36].

\subsubsection{Total Iron}

Iron is an essential element for almost all living organisms. The average adult human being stores about 1 - $3 \mathrm{~g}$ of iron in his or her body [37]. Iron is also a heavy metal which is not biologically degradable unlike most organic pollutants and poses health risk to the consumer at high concentrations [34] [38]. The within sample variability of total iron in the various raw and tap water sources were slightly high i.e. raw water $(\mathrm{CV}=11.75-73.01 \%)$, tap water $(\mathrm{CV}=24.86 \%$ $66.75 \%)$ as well as the overall raw $(\mathrm{CV}=124.22 \%)$ and tap $(\mathrm{CV}=53.54 \%)$ water sources. Total iron exhibited significant variations $(\mathrm{p}<0.05)$ among the various raw and tap water sources. Surface well below Nyandozo Primary School registered the highest total iron $(0.3183 \mathrm{mg} / \mathrm{L})$ while the Protected spring below Kikuba Hill had the least total iron content $(0.0340 \mathrm{mg} / \mathrm{L})$. The tap water near Bassajjabalaba Primary School recorded the highest total iron $(0.136 \mathrm{mg} / \mathrm{L})$ and that at Kanyamabona Trading Centre had the least iron content $(0.036 \mathrm{mg} / \mathrm{L})$. On the other hand, there was no significant variation $(p>0.05)$ in the total iron in the raw and tap water sources on overall much as raw water $(0.0924 \mathrm{mg} / \mathrm{L})$ recorded slightly higher total iron than tap water $(0.0907 \mathrm{mg} / \mathrm{L})$.

All the raw drinking water sources had total iron concentrations within the UNBS acceptable value $(<1 \mathrm{mg} / \mathrm{L})$. Only the surface well below Nyandozo Primary School had slightly higher total iron content than the standard WHO value 
$(<0.3 \mathrm{mg} / \mathrm{L})$. The rest of the raw water sources had total iron concentrations within the WHO guideline value. Tap water from all the selected sites (Table 2) had total iron contents within the UNBS and WHO guidelines of $<0.2 \mathrm{mg} / \mathrm{L}$ and $<0.3 \mathrm{mg} / \mathrm{L}$ respectively. Similarly, the overall raw and tap water sources (Table 3) contained total iron concentrations within the accepted UNBS and WHO standards. The high iron concentrations in the Surface well below Nyandozo Primary School could be associated with sewage runoff from the latrines and landfills (waste sites) in the school especially during rainfall. There is also a possibility of direct ground seepage of iron from the latrines into the surface well. According to Water Stewardship Information Series [39], sewage and landfill leachate are sources of iron to local groundwater.

However, the high total iron in the tap water near Bassajjabalaba Primary School can be associated with the high corrosion of metal pipes under acidic $\mathrm{pH}$ conditions. On the other hand the low iron content in the tap water at Kanyamabona Trading Centre is due to reduced corrosion of the metal pipes under neutral water conditions. This is further supported by the significant negative correlation between total iron and $\mathrm{pH}(\mathrm{r}=-0.45, \mathrm{p}<0.01)$ of tap water (Table 4). Rahmanian et al. [28] asserts that acidic water corrodes metal pipes and plumping system.

\subsubsection{Residual Alum}

Aluminium or iron salts (granules, powders or tablets) added to water, coagulate and flocculate impurities and promote rapid and efficient sedimentation and also enable the inactivation of microbes by free chlorine [23]. The aluminium forms aluminosilicates which frequently deposit onto plumbing materials in distribution systems thus protecting the pipes from corrosion [40]. The residual alum in the tap water sources exhibited relatively moderate variability within the water samples $(\mathrm{CV}=22.81 \%-40.63 \%)$ with significant variations $(\mathrm{p}<0.05)$ of the mean residual alum among the various tap water sources. Tap water near Bushenyi Town School had the highest mean residual alum $(0.113 \mathrm{mg} / \mathrm{L})$ while the tap water at Kanyamabona Trading Centre recorded the least residual alum $(0.092 \mathrm{mg} / \mathrm{L})$. The overall mean residual alum was $0.103 \mathrm{mg} / \mathrm{L}$.

The amounts of the residual alum in the tap water sources were within the UNBS and WHO standard of $<0.2 \mathrm{mg} / \mathrm{L}$. The mean residual alum for tap water was within the WHO recommended range for safe domestic potable water attributable to the efficient water treatment process that uses proportionate doses of these chemicals. The high residual alum in the tap water near Bushenyi Town School can also be associated with the increased corrosion of the metal pipes under acidic $\mathrm{pH}$ conditions (Table 2) to release aluminium into the water. Conversely, the low residual alum in the tap water at Kanyamabona Trading Centre is due to reduced corrosion of the metal pipes under neutral water conditions (Table 2). This is also in line with the fact that acidic water leaches metals from pipes [28] [33] as opposed to neutral and alkaline waters. The significant negative correlation between residual alum and $\mathrm{pH}(\mathrm{r}=-0.22, \mathrm{p}<0.01)$ in tap water 
(Table 4) further justifies the corrosion of alum under acidic conditions.

\subsubsection{Free Residual Chlorine}

Disinfection of water of pathogenic microorganisms is a necessary requirement in the supply of safe drinking-water. It commonly involves the use of reactive chemicals like chlorine [23]. However, there have been concerns about the formation of toxic and carcinogenic by-products of chlorination [41]. Therefore, chlorine should be added to the water in a way that its residue remains are within acceptable limits so as to prevent any unpredicted pollution. There were significant variations $(\mathrm{p}<0.05)$ of free residual chlorine in the tap water sources with relatively low variability within the samples $(\mathrm{CV}=8.90 \%-38.98 \%)$. The highest mean free residual chlorine $(0.275 \mathrm{mg} / \mathrm{L})$ was recorded in the tap water near Bassajjabalaba Primary School and the lowest in tap water at Kanyamabona Trading Centre $(0.192 \mathrm{mg} / \mathrm{L})$. The overall mean free residual chlorine was 0.244 $\mathrm{mg} / \mathrm{L}$. The mean free residual chlorine in most of the selected tap water sources were within the range of UNBS and WHO guideline value of $0.2-0.5 \mathrm{mg} / \mathrm{L}$. However, the free residual chlorine in tap water at Kanyamabona Trading Centre was slightly below the lower limit of UNBS and WHO standards. The mean free residual chlorine in the tap water sources were within the recommended ranges for safe domestic potable water also attributable to the efficient water treatment process that uses proportionate doses of these chemicals. According to WHO [23], $\mathrm{pH}$ control is necessary at all stages of water treatment to ensure satisfactory water clarification and disinfection. For effective disinfection with chlorine, the $\mathrm{pH}$ should preferably be less than 8 . Hence the mean $\mathrm{pH}$ range for tap water sources of 5.68 - 7.06 was suitable for chlorine disinfection. This is also further supported by the significant negative correlation between $\mathrm{pH}$ and free residual chlorine $(\mathrm{r}=-0.56, \mathrm{p}<0.01)$ in tap water $($ Table 4$)$.

\subsection{Faecal Coliforms in the Raw and Tap Water Sources in Bushenyi Municipality}

The coliform bacterium is a primary bacterial indicator for faecal pollution in water [42] [43]. Hence bacterial total coliform and Escherichia coli (E. coli) examination provide indication of the hygienic condition of drinking water and are major tools in the assessment of the health risk posed by pathogen in water [44]. The faecal coliforms exhibited high variability within samples of the raw water sources $(\mathrm{CV}=55.87 \%-167.94 \%)$ as shown in Table 1 . Faecal coliforms were only found in tap water at Kanyamabona Trading Centre (Table 2) with a very high within sample variability $(\mathrm{CV}=175.41 \%)$. Similarly the variability of faecal coliforms was very high within samples of raw (CV = 188.26\%) and tap $(\mathrm{CV}=436.98 \%)$ water sources on overall (Table 3$)$. Significant variations $(\mathrm{p}<$ 0.05 ) were recorded in the mean faecal coliform counts in the various raw water sources as well as between the raw and tap water sources. The borehole below Bweranyangi Junior School had the highest mean faecal coliforms (16.83 $\mathrm{CFU} / 100 \mathrm{ml}$ ) while the Protected spring below Kikuba Hill had the least mean 
coliform counts $(0.40 \mathrm{CFU} / 100 \mathrm{ml})$. Raw water $(7.07 \mathrm{CFU} / 100 \mathrm{ml})$ sources recorded higher mean faecal coliform counts than tap water $(0.05 \mathrm{CFU} / 100 \mathrm{ml})$ sources (Table 3). The protected springs below Kyeitembe Vocational Secondary School and Kikuba Hill had faecal coliforms within the UNBS and WHO guideline value ( $\leq 3 \mathrm{CFU} / 100 \mathrm{ml}$ ). On the other hand, faecal coliform amounts in the borehole below Bweranyangi Junior School, surface well below Nyandozo Primary School and surface well below Kyeitembe Trading Centre were above the UNBS and WHO standard faecal coliform counts. The mean faecal coliforms $(0.25 \mathrm{CFU} / 100 \mathrm{ml})$ in the tap water at Kanyamabona Trading Centre were beyond the UNBS and WHO acceptable count in tap water $(0 \mathrm{CFU} / 100 \mathrm{ml})$. Similarly the overall mean faecal coliform counts in the raw and tap water sources were above the UNBS and WHO standard values for raw and tap water (Table 3).

The presence of the faecal coliforms in the borehole below Bweranyangi Junior School in Kacuncu, surface well below Nyandozo Primary School and surface well below Kyeitembe Trading Centre as well as the tap water at Kanyamabona Trading Centre beyond the UNBS and WHO standards suggests sewage contamination of these water sources. Humans drinking such water untreated are at a risk of waterborne diseases. The high faecal coliform counts in the water sources around the schools and the trading centre could be attributed to the dense human population and generally poor sanitation. Pit latrines are also the major waste facility used in these areas. UBOS [13] also indicated that some households do not have toilet facilities in Ishaka $(0.3 \%)$, Central $(0.4 \%)$ and Nyakabirizi $(0.4 \%)$ divisions of the municipality. Hence the microbial load may also be attributed to sub-surface leaching from pit latrines and washing of faecal material from open defecation into the water sources during heavy rainfall. Pit latrines are sources of bacteria to groundwater and the microbial (bacterial) load into springs in Katwe and Kisenyi parishes, Kampala city, Uganda were also attributed to sub-surface leaching from pit latrines [45] [46]. The sampling period of September to October 2016 also coincided with periods of peak rains (September to December) in Bushenyi district which flood the wetlands [8]. Several researchers [47] [48] have associated rainfall events with high E. coli counts. The overall significant positive correlation between faecal coliforms and turbidity ( $\mathrm{r}$ $=0.11, \mathrm{p}<0.05)$ points to the transportation of the coliforms into the surface water with particulate matter (responsible for turbidity) from the land surface. The very high $E$. coli in Nyaruzinga wetland water near; sewage discharge grounds (3000 CFU/100 ml), fish ponds (258.3 CFU/100 ml), dip tank (613.3 $\mathrm{CFU} / 100 \mathrm{ml})$, raw water reservoir $(96.7 \mathrm{CFU} / 100 \mathrm{ml})$ and car washing bay $(75.0$ $\mathrm{CFU} / 100 \mathrm{ml}$ ) [26] further support the faecal contamination of the wetland water.

In addition to the filtration of contaminated water by soil in a protected spring, the low faecal coliforms in the protected spring below Kikuba Hill compared to other water sources could be associated with the relatively more acidic water conditions $(\mathrm{pH}=5.21)$ as opposed to other water sources. This is justified 
by the overall significant positive correlation between $\mathrm{pH}$ and faecal coliform counts $(\mathrm{r}=0.26, \mathrm{p}<0.00)$. E. coli counts increase with increase in $\mathrm{pH}$ within the suitable $\mathrm{pH}$ range for its survival. They decrease below $\mathrm{pH} 5$ and beyond $\mathrm{pH} 9$ [44] [49] [50]. However the mean range of $\mathrm{pH}$ of all the water sources (5.21 7.06) was within the suitable range for E. coli.

The presence of faecal coliforms in tap water in one of the sampling sites in Kanyamabona trading centre is not expected under normal circumstances. This is probably attributed to leakage of faecal matter from Kanyamabona village into corroded metallic water pipes or leakage through low density polyethylene plastic pipes. According to WHO [23], lower $\mathrm{pH}$ water (approximate $\mathrm{pH} 7$ or less) is more likely to be corrosive to pipes in household water systems. With the mean $\mathrm{pH}$ for tap water sources in the present study ranging from $5.68-7.06$ and that in the tap water at Kanyamabona Trading Centre being 7.06, cases of corrosion of pipes are expected. Corrosion of metallic pipes in the supply of water has been reported by Heathcote [51]. Hence failure to minimize corrosion can result in the contamination of drinking-water [23]. Furthermore, the lower free residual chlorine in tap water at Kanyamabona Trading Centre, Ishaka division than the UNBS and WHO standards subjects the water to bacterial contamination. WHO [23] asserted that a sudden disappearance of stable residual chlorine causes contamination of water and failure to maintain residual chlorine at points in a distribution system promotes growth of bacteria. This is further supported by the significant negative correlation between faecal coliforms and free residual chlorine $(\mathrm{r}=-0.75, \mathrm{p}<0.01)$ in tap water. Safari et al. [26] also recorded E. coli in treated tap water $(1.7 \mathrm{CFU} / 100 \mathrm{ml})$ from Bushenyi town pointing to contamination of the tap water by bacteria.

\section{Conclusions}

The quality of tap water within the municipality tapped from Nyaruzinga wetland was better than the raw drinking water sources (borehole, springs, wells) from the same wetland. Hence the use of piped tap water by the urban inhabitants as opposed to raw water sources needs to be promoted to increase access to safe water. The high total iron (heavy metal) in the surface well below Nyandozo Primary School in Kacuncu should be checked to avoid toxicity of the metal to the users of the water.

The quality of tap water in the three divisions of Bushenyi Municipality was generally suitable for domestic use except that from Kanyamabona trading centre, Ishaka Division which recorded some faecal contamination. Thus there is need for constant surveillance of the water pipes by National Water and Sewerage Corporation (NWSC) so as to identify, repair or replace worn out and corroded pipes to avoid any contamination.

National Water and Sewerage Corporation should ensure adequate chlorination of the water at the treatment plant so as to eliminate bacteria from thriving in the tap water, a case of tap water at Kanyamabona Trading Centre, Ishaka di- 
vision.

Boiling or treatment with hypochlorite solution of water from most of the raw water sources including tap water from Kanyamabona trading centre, Ishaka Division is recommended to kill the bacterial parasites before drinking.

\section{Acknowledgements}

The authors are grateful to the Department of Biology, Mbarara University of Science and Technology (MUST) and the staff of Nyaruzinga water treatment plant for offering facilities to undertake this research. Most importantly, the authors give thanks to God Almighty, the Giver and Sustainer of life.

\section{References}

[1] WHO and UNCF (2000) Global Water Supply and Sanitation Assessment 2000 Report. World Health Organization and United Nations Children's Fund, Geneva. http://www.who.int/water_sanitation_health/monitoring/jmp2000.pdf

[2] Agnew, C. and Woodhouse, P. (2011) Water Resources and Development. Routledge, London and New York.

[3] Oyem, H.H., Oyem, I.M. and Ezeweali, D. (2014) Temperature, pH, Electrical Conductivity, Total Dissolved Solids and Chemical Oxygen Demand of Groundwater in Boji-Boji Agbor/Owa Area and Immediate Suburbs. Research Journal of Environmental Sciences, 8, 444-450. https://doi.org/10.3923/rjes.2014.444.450

[4] Naiga, R., Penker, M. and Hogl, K. (2015) Challenging Pathways to Safe Water Access in Rural Uganda: From Supply to Demand-Driven Water Governance. International Journal of the Commons, 9, 237-260. https://doi.org/10.18352/ijc.480

[5] Mathew, B. (2004) Ensuring Sustained Beneficial Outcomes for Water and Sanitation Programmes in the Developing World. PhD Thesis, Cranfield University, Institute of Water and Environment.

[6] DWD (2011) Assessment of the Effectiveness of the Community-Based Maintenance System for Rural Water Supply Facilities Report. Directorate of Water Development, Ministry of Water and Environment, Kampala.

[7] UBOS (2011) Statistical Abstract 2011. Uganda Bureau of Statistics (UBOS), Entebbe.

[8] Mugyenyi, C. and Mujurizi, J. (2000) Bushenyi. National Wetlands Programme Wetland Inspection Division, Ministry of Water, Lands and Environment, Kampala.

[9] Kayombo, S. and Jorgensen, S.E. (2006) Lake Victoria. In: Jorgensen, S.E., Ed., EXperiences and Lessons Learned, Royal Danish University of Pharmaceutical Sciences, Copenhagen.

[10] NEMA (2010) The State of the Environment Report for Uganda 2010. National Environment Management Authority (NEMA), Kampala.

[11] DWD (2017) Ministry of Water and Environment. Uganda Water Supply Atlas, Bushenyi District.

[12] NWSC (2014) Area Switch Program for Bushenyi Area for Financial Year 2014/15.

[13] UBOS (2017) The National Population and Housing Census 2014-Area Specific Profile. Kampala.

http://www.ubos.org/onlinefiles/uploads/ubos/2014CensusProfiles/BUSHENYI.pdf 
[14] Ministry of Education and Sports (2014) Environmental and Social Management Framework for Skills Development Project (SDP). Final Version. http://documents.worldbank.org/curated/pt/444581468103474647/pdf/E47410AFR 0EA0P0Box385433B00PUBLIC0.pdf

[15] Nagudi, B. (2011) Status of Geological Resources in Uganda, for the Embassy of the Republic of Korea in Uganda.

[16] APHA (1998) Standard Methods for the Examination of Water and Waste Water. American Public Health Association, 874 p.

[17] APHA, AWWA, and WEF (2005) Standard Methods for the Examination of Water and Wastewater. 21st Edition, American Public Health Association, Washington DC.

[18] AOAC (2002) Physico-Chemical Water Quality Analysis. In: Official Methods of Analysis, Volume 1, AOAC International, Washington DC.

[19] NWSC (2015) Standard Operating Procedures for Routine Water Quality Monitoring. Standards Operations Manual.

[20] Reda, A.H. (2016) Physico-Chemical Analysis of Drinking Water Quality of Arbaminch Town. Journal of Environmental \& Analytical Toxicology, 6, 356. https://doi.org/10.4172/2161-0525.1000356

[21] Palamuleni, L. and Akoth, M. (2015) Physico-Chemical and Microbial Analysis of Selected Borehole Water in Mahikeng, South Africa. Journal of Environmental Research and Public Health, 12, 8619-8630. https://doi.org/10.3390/ijerph120808619

[22] Trivede, P., Bajpai, A. and Thareja, S. (2010) Comparative Study of Seasonal Variations in Physico-Chemical Characteristics in Drinking Water Quality of Kanpur, India with Reference to 200 MLD Filtration Plant and Groundwater. Natural Science, 8, 11-17.

[23] WHO (2011) Guidelines for Drinking-Water Quality. 4th Edition, World Health Organization Press Geneva. http://apps.who.int/iris/bitstream/10665/44584/1/9789241548151_eng.pdf

[24] UNBS (2008) Drinking (Potable) Water Quality Specifications. 2nd Edition, Uganda National Bureau of Standards, Kampala.

[25] Bot, A. and Benites, J. (2005) The Importance of Soil Organic Matter Key to Drought-Resistant Soil and Sustained Food and Production. FAO Soils Bulletin 80, FAO, Rome.

[26] Safari, D., Mulongo, G., Byarugaba, D. and Tumwesigye, W. (2012) Impact of Human Activities on the Quality of Water in Nyaruzinga Wetland of Bushenyi District Uganda. International Research Journal of Environmental Sciences, 1, 1-6.

[27] Vyas, V.G., Hassan, M.M., Vindhani, S.I., Parmar, H.J. and Bhalani, V.M. (2015) Physicochemical and Microbiological Assessment of Drinking Water from Different Sources in Junagadh City, India. American Journal of Microbiological Research, 3, 148-154.

[28] Rahmanian, N., Ali, S.H.B., Homayoonfard, M., Ali, N.J., Rehan, M., Sadef, Y. and Nizami, A.S. (2015) Analysis of Physiochemical Parameters to Evaluate the Drinking Water Quality in the State of Perak, Malaysia. Journal of Chemistry, 2015, Article ID: 716125.

[29] Kavcar, P., Sofuoglu, A. and Sofuoglu, S.C. (2009) A Health Risk Assessment for Exposure to Trace Metals via Drinking Water Ingestion Pathway. International Journal of Hygiene and Environmental Health, 212, 216-227. https://doi.org/10.1016/j.ijheh.2008.05.002 
[30] Cidu, R., Frau, F. and Tore, P. (2011) Drinking Water Quality: Comparing Inorganic Components in Bottled Water and Italian Tap Water. Journal of Food Composition and Analysis, 24, 184-193. https://doi.org/10.1016/j.jfca.2010.08.005

[31] Muhammad, S., Shah, M.T. and Khan, S. (2011) Health Risk Assessment of Heavy Metals and Their Source Apportionment in Drinking Water of Kohistan Region, Northern Pakistan. Microchemical Journal, 98, 334-343. https://doi.org/10.1016/j.microc.2011.03.003

[32] Khan, S., Shahnaz, M., Jehan, N., Rehman, S., Shah, M.T. and Din, I. (2013) Drinking Water Quality and Human Health Risk in Charsadda District, Pakistan. Journal of Cleaner Production, 60, 93-101. https://doi.org/10.1016/j.jclepro.2012.02.016

[33] WHO (2010) Guideline for Drinking Water Quality. 3rd Edition, World Health Organization, Geneva.

[34] Yasin, M., Ketema, T. and Bacha, K. (2015) Physico-Chemical and Bacteriological Quality of Drinking Water of Different Sources, Jimma Zone, Southwest Ethiopia. BMC Research Notes, 8, 541. https://doi.org/10.1186/s13104-015-1376-5

[35] Walakira, P. (2011) Impact of Industrial Effluents on Water Quality of Receiving Streams in Nakawa-Ntinda, Uganda. MSc Thesis (Unpubl.), Makerere University, Kampala, 58.

https://news.mak.ac.ug/documents/Makfiles/theses/Walakira_Paul.pdf

[36] Durfor, C.N. and Becker, E. (1964) Public Water Supplies of the 100 Largest Cities in the United States. In: Geological Survey Water-Supply, US Government Printing Office, Washington, Vol. 1812, 364.

[37] Abbaspour, N., Hurrell, R. and Kelishadi, R. (2014) Review on Iron and Its Importance for Human Health. Journal of Research in Medical Sciences, 19, 164-174.

[38] WHO (2003) Technical Support Document for Ontario Drinking Water Standards, Objectives and Guidelines. Ministry of the Environment.

http://www.ene.gov.on.ca/stdprodconsume/groups/lr/@ene/@resources/documents /resource/std01_079707.pdf

[39] Water Stewardship Information Series (2007) Iron \& Manganese in Ground Water. http://www.rdn.bc.ca/cms/wpattachments/wpID2284atID3808.pdf

[40] APHA (2006) Standard Methods for the Examination of Water and Wastewater. 21st Edition, APHA, AWWA, WPCF, Washington DC.

[41] Walker, B.H., Conway-Schemp, N. and Krofchik, M. (1994) Risks Associated with Chlorination. Carnegie Mellon University, Engineering Design Research Center. http://repository.cmu.edu/cgi/viewcontent.cgi? article $=1060 \&$ context $=$ cee

[42] Parihar, S.S., Kumar, A., Kumar, A., Gupta, R.N., Pathak, M., Shrivastav, A. and Pandey, A.C. (2012) Physico-Chemical and Microbiological Analysis of Underground Water in and around Gwalior City, MP, India. Research Journal of Recent Science, 1, 62-65.

[43] Mohan, D., Gaur, A. and Chodhary, D. (2007) Study of Limnology and Microbiology of NayaTalab, Jodhpur, Rajasthan. Proceedings of National Symposium on Limnology, Udaipur, 19-21 February 2007, 64-68.

[44] Lukubye, B. and Andama, M. (2017) Bacterial Analysis of Selected Drinking Water Sources in Mbarara Municipality, Uganda. Journal of Water Resource and Protection, 9, 999-1013. https://doi.org/10.4236/jwarp.2017.98066

[45] Bloodless, D., Zvikomborero, H., David, L. and Edward, G. (2006) Assessment of the Impacts of Pit Latrines on Groundwater Quality in Rural Areas: A Case Study from Marondera District, Zimbabwe. Physics and Chemistry of the Earth, 31, 
779-788. https://doi.org/10.1016/j.pce.2006.08.031

[46] Haruna, R., Ejobi, F. and Kabagambe, E.K. (2005) The Quality of Water from Protected Springs in Katwe and Kisenyi Parishes, Kampala City, Uganda. African Health Sciences, 5, 14-20.

[47] Nsubuga, F.B., Kansiime, F. and Okot, O.J. (2004) Pollution of Protected Springs in relation to High and Low Density Settlements in Kampala, Uganda. Physics and Chemistry of the Earth, 29, 1153-1159. https://doi.org/10.1016/j.pce.2004.09.001

[48] Howard, G., Pedley, S., Barett, M., Nalubega, M. and Johal, K. (2003) Risk Factors Contributing to Microbiological Contamination of Shallow Groundwater in Kampala, Uganda. Water Research, 37, 3421-3429. https://doi.org/10.1016/S0043-1354(03)00235-5

[49] Karak, T. and Bhattacharyya, P. (2011) Human Urine as a Source of Alternative Natural Fertilizer in Agriculture: A Flight of Fancy or an Achievable Reality. Resources, Conservation and Recycling, 55, 400-408. https://doi.org/10.1016/j.resconrec.2010.12.008

[50] Buchanan, R.L. and Edelson, S.G. (1999) Effect of pH-Dependent, Stationary Phase Acid Resistance on the Thermal Tolerance of Escherichia coli O157:H7. Food Microbiology, 16, 447-458. https://doi.org/10.1006/fmic.1998.0260

[51] Heathcote, M. (2009) Plastics Pipe in Water and Waste Water Infrastructure. General Technical Information. Plastics Industry Pipe Association of Australia Limited. http://www.pipa.com.au/sites/default/files/document/attachment/tp004.pdf 